\title{
A Fast and Robust Technique for 3D-2D Registration of CT to Single Plane X-Ray Fluoroscopy
}

\author{
Md. Nazmul Haque ${ }^{\mathrm{a}}$, Mark R. Pickeringa ${ }^{\mathrm{a}}$, Abdullah Al Muhit ${ }^{\mathrm{a}}$, Michael R. Frater ${ }^{\mathrm{a}}$, Jennie M. Scarvell ${ }^{\mathrm{b}}$, Paul N. Smith ${ }^{\mathrm{b}}$ \\ ${ }^{a}$ School of Engineering and Information Technology, UNSW Canberra, Canberra, Australia \\ ${ }^{b}$ The Trauma and Orthopaedic Research Unit, The Canberra Hospital, Canberra, Australia
}

\begin{abstract}
The application of 3D-2D image registration can be enormously helpful for different clinical purposes, such as image-guided surgery and the kinematic analysis of bones in knee and ankle joints. A limitation of this approach is the need to recalculate the voxel values in the 3D volume for every iteration of the registration procedure prior to generating a digitally reconstructed radiograph (DRR). In this paper we propose a new multi-phase 3D-2D image registration algorithm which employs partial 3D volumes to estimate outof-plane rotations. In our proposed algorithm only one full 3-D update is used to generate a 2D projection during the registration procedure. Experimental results show that our proposed method can provide similar registration accuracy to the commonly used approach which employs 3-D updates at every iteration. As a result of reducing the number of 3-D updates, the proposed approach reduces the time required to perform the registration by a factor of 10 to 20 without any accompanying loss of registration accuracy.
\end{abstract}

Keywords: 3D-2D image registration, multi-modal similarity measure, image guided surgery, kinematic analysis.

\section{Introduction}

In recent years 3D-2D image registration has become an essential technique for image-guided surgery, medical diagnosis, treatment planning, and monitoring the progress of rehabilitation. However, the computational complexity of this registration technique is still a challenge. The goal of any 3D-2D image registration technique is to find the geometric transformation which spatially aligns the $2 \mathrm{D}$ projection of a $3 \mathrm{D}$ volume and a 2D image. This transformation is typically estimated by comparing a digitally reconstructed radiograph (DRR) generated from a $3 \mathrm{D}$ volume with a $2 \mathrm{D}$ image. In clinical applications, the 3D volume is usually captured using Computed Tomography (CT) or Magnetic Resonance Imaging (MRI) and the 2D image is a single $\mathrm{x}$-ray or a frame from a fluoroscopy video.

Different feature and intensity-based techniques have been used for 3D-2D medical image registration (Markelj et al., 2012). In feature based approaches, a set of image features such as corners, points or more complex structures are located in the images to be registered and parameters which produce the best correspondence between the two sets of features are estimated. The contour and point-based techniques presented in (Zuffi et al., 1999; Yamazaki et al., 2004) are examples of this strategy. On the other hand, intensity based methods try to find a spatial correspondence by comparing image intensity values (Mahfouz et al., 2003; Weese et al., 1997b; Penney et al., 1998; Tomazevic et al., 2003). The two primary components of intensity based registration approaches are: the similarity measure and the optimization procedure (Pickering et al., 2009; Maes et al., 1999). The similarity measure quantifies the amount of correspondence between two images and indicates the success or convergence of the matching process. The similarity measure must be multi-modal when the images to be registered are from dissimilar sensors, as is the case in many medical imaging applications.

A wide range of multi-modal similarity measures have been proposed in the literature for medical image registration (Weese et al., 1997b; Penney et al., 1998; Tomazevic et al., 2003; Pluim et al., 2003). Mutual information (MI) based similarity measures have been widely used in different medical image registration applications (Pluim et al., 2003; Markelj et al., 2008). In (Zollei et al., 2001), the authors used mutual information and sparse histograms to estimate the probability of image intensities. In (Wang and Vemuri, 2007), a new multimodal similarity measure known as the Cross-Cumulative Residual Entropy (CCRE) was proposed. CCRE has been shown to provide faster convergence to the global maximum than $\mathrm{MI}$ and also a greater range of initial disparities for which successful convergence can be obtained (Wang and Vemuri, 2007).

A new multimodal similarity measure called the Sum of Conditional Variances (SCV) was proposed in (Pickering et al., 2009). Unlike existing multi-modal similarity measures, SCV allows the gradient vector and Hessian matrix required for standard non-linear least squares approaches to be calculated using the same procedure as that used for the sum-of-squared difference (SSD). The SCV based measure has been shown to provide better registration accuracy when compared with CCRE based techniques (Pickering et al., 2009).

The optimization procedure automatically determines the best spatial alignment between the source and target data. The optimization procedures used in the literature for 3D-2D image registrations are diverse and can be broadly categorized into non-gradient and gradient based methods. In literature, registration approaches those use non-gradient based optimization 
technique can be found in (Mahfouz et al., 2003; Weese et al., 1997b; de Bruin et al., 2008). The most commonly used gradient based approaches are the Gauss-Newton and LevenbergMarquardt algorithms (Maes et al., 1999; Lavallee and Szeliski, 1995; Livyatan et al., 2003). In this paper, SCV and GaussNewton optimization are adopted in the proposed fast registration algorithm.

Most 3D CT to 2D fluoroscopy registration techniques iteratively update the position of the 3D volume prior to DRR generation. These DRR images are then compared with the fluoroscopy frames to calculate the similarity measure (Tomazevic et al., 2003; Russakoff et al., 2005; Weese et al., 1999). Updating the $3 \mathrm{D}$ volume at every iteration is the most computationally expensive part of the $3 \mathrm{D}-2 \mathrm{D}$ registration process.

To reduce the computational complexity of the 3D-2D registration process, several techniques have been proposed in the literature. Approaches based on efficient DRR generation have been proposed in (Weese et al., 1997b; Lacroute and Levoy, 1994; Birkfellner et al., 2003, 2005). However, these approaches must still employ $3 \mathrm{D}$ volume updates at every iteration of the iterative process prior to the efficient DRR generation.

In (Jans et al., 2006; Lujan et al., 1998) a number of DRRs are generated offline for a set of predefined out-of-plane rotations and these DRRs are then used as a reference to estimate out-of-plane rotations during the registration process. These offline DRR generation approaches can speed up the registration process but incur the penalty of reduced accuracy in estimating out-of-plane transform parameters.

In (Weese et al., 1997a), for a particular application, a fast 3D-2D image registration technique using a small part of the CT image showing only the vertebra was proposed. However, this approach requires a manual segmentation process to segment the vertebra and also requires 3D updates of the segmented volume at every iteration.

In (Birkfellner et al., 2003) the authors reduce the computational complexity of 3D-2D registration by decoupling the inplane rotation $\left(R_{z}\right)$ from the other 5 rigid body transform parameters. In their approach, the full $3 \mathrm{D}$ volumes still need to be positioned at the new estimated position during the iterative process prior to DRR generation and decoupling of the in-plane rotation.

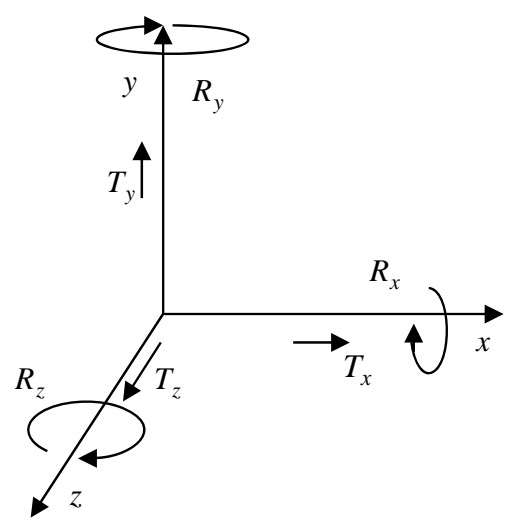

Figure 1: The orientation of the 6 parameters that are used to describe $3 \mathrm{D}$ rigid body motion
A number of fast bi-planar or multi-planar based approaches have also been proposed in the literature (Chen et al., 2008; Fu and Kuduvalli, 2008) which allow the 3D rigid-body parameters to be estimated very precisely. These techniques have the advantage of more 2D information to correctly determine the true $3 \mathrm{D}$ pose of the anatomical structure but also incur the problem of requiring multiple fluoroscopy units to acquire the extra images which is costly and not normally required for standard medical imaging procedures. This setup also limits the field of view of the images where the x-ray sources intersect and requires multiple doses of radiation for each patient.

As the algorithms mentioned in literature always incorporate $3 \mathrm{D}$ volume updates and incur the cost of computational complexity at the iterative process, here, to reduce the computational complexity related to volume updates, we propose a new fast single-plane 3D-2D image registration algorithm which processes most of the iterative part of the algorithm using 2D information instead of updating the 3D CT volume. The proposed algorithm estimates in-plane parameters by comparing the DRR generated at the original 3D CT position with the $\mathrm{X}$-ray fluoroscopy image during the iterative process. This eliminates full 3D volume updates during the optimization and hence DRR generation is also not required for estimating the inplane parameters. The proposed algorithm utilizes an efficient way of calculating out-of-plane rotations by updating partial 3D data instead of updating the full 3D volume. The proposed algorithm estimates out-of-plane translation by updating the 2D DRR instead of updating the 3D CT volume.

The remainder of this paper is organized as follows: Section II presents an overview of 3D-2D registration. A description of the proposed fast algorithm is provided in section III. In section IV the registration accuracy, speed and convergence rate of the proposed algorithm are compared to the alternative approach which updates the full 3D volume at every iteration. Section V contains our conclusions from these results.

\section{3D-2D IMAGE REGISTRATION}

In image registration techniques, when the similarity between the source and target images is maximized the images are considered to be spatially aligned, or registered, with each other. In the case of $3 \mathrm{D}-2 \mathrm{D}$ registration, this problem is more difficult as there are 6 parameters that can be varied to describe the $3 \mathrm{D}$ rigid-body motion. These six parameters are shown in Figure. 1. They are: translation in the $x, y$ and $z$ directions $\left(T_{x}, T_{y}\right.$ and $\left.T_{z}\right)$ and rotations about the $x, y$ and $z$ axes $\left(R_{x}, R_{y}\right.$ and $R_{z}$ ). Based on the rigid body motion model, the geometric transformation matrix for translations is defined as follows:

$$
\left[\begin{array}{cccc}
1 & 0 & 0 & T_{x} \\
0 & 1 & 0 & T_{y} \\
0 & 0 & 1 & T_{z} \\
0 & 0 & 0 & 1
\end{array}\right]\left[\begin{array}{c}
x \\
y \\
z \\
1
\end{array}\right]=\left[\begin{array}{c}
x^{\prime} \\
y^{\prime} \\
z^{\prime} \\
1
\end{array}\right]
$$

where $(x, y, z)$ are the original coordinates of the voxel and $\left(x^{\prime}, y^{\prime}, z^{\prime}\right)$ are the transformed coordinates. The transformation matrices for rigid body rotations of $\theta$ around the $x, y$ and $z$ axes 
are given in (2), (3) and (4) respectively.

$$
\begin{aligned}
& {\left[\begin{array}{cccc}
1 & 0 & 0 & 0 \\
0 & \cos \theta & -\sin \theta & 0 \\
0 & \sin \theta & \cos \theta & 0 \\
0 & 0 & 0 & 1
\end{array}\right]\left[\begin{array}{l}
x \\
y \\
z \\
1
\end{array}\right]=\left[\begin{array}{c}
x^{\prime} \\
y^{\prime} \\
z^{\prime} \\
1
\end{array}\right]} \\
& {\left[\begin{array}{cccc}
\cos \theta & 0 & \sin \theta & 0 \\
0 & 1 & 0 & 0 \\
-\sin \theta & 0 & \cos \theta & 0 \\
0 & 0 & 0 & 1
\end{array}\right]\left[\begin{array}{l}
x \\
y \\
z \\
1
\end{array}\right]=\left[\begin{array}{c}
x^{\prime} \\
y^{\prime} \\
z^{\prime} \\
1
\end{array}\right]} \\
& {\left[\begin{array}{cccc}
\cos \theta & -\sin \theta & 0 & 0 \\
\sin \theta & \cos \theta & 0 & 0 \\
0 & 0 & 1 & 0 \\
0 & 0 & 0 & 1
\end{array}\right]\left[\begin{array}{l}
x \\
y \\
z \\
1
\end{array}\right]=\left[\begin{array}{c}
x^{\prime} \\
y^{\prime} \\
z^{\prime} \\
1
\end{array}\right]}
\end{aligned}
$$

For every iteration of the registration process a 3D rigid body geometric transformation matrix is calculated by multiplying the individual translation and rotation matrices from equations (1), (2), (3) and (4). So the total transformation matrix can be modeled as follows:

$$
F=T R
$$

where $T$ is the translation matrix and $R$ is the rotation matrix for the combined rotations around the $x, y$ and $z$ axes. This transformation matrix $F$ is applied to the $3 \mathrm{D}$ volume to produce a change in the $3 \mathrm{D}$ position of the object and the updated $3 \mathrm{D}$ volume is reduced to a 2D DRR. The similarity measure is then calculated for the DRR and X-ray fluoroscopy images. As mentioned before different similarity measure techniques have been used in literature to measure the similarity between target and reference image. In case of SCV, let $I\left(x_{i}^{\prime}, y_{i}^{\prime}\right)$ be the DRR image that is to be registered and $P\left(x_{i}, y_{i}\right)$ be the reference X-ray fluoroscopy image. The coordinates $\left(x_{i}^{\prime}, y_{i}^{\prime}\right)$ and $\left(x_{i}, y_{i}\right)$ denote the locations of the pixels in $I$ and $P$ respectively for $i=1 \ldots \ldots . . N$ where $N$ is the number of pixels in the image. The similarity measure for image $I$ and $P$ is given by

$$
S(\mathrm{~m})=\sum_{j} E\left(\left[I_{i}-E\left(I_{i} \mid P_{i} \in \Delta_{j}\right)\right]^{2} \mid I_{i} \in \Delta_{j}\right)
$$

where $E()$ denotes the expectation operator, $\Delta_{j}$ denotes a set of histogram bins that span the range of values in $P$ and $\mathrm{m}$ is the vector of motion parameters $\left[T_{x} T_{y} T_{z} R_{x} R_{y} R_{z}\right]^{T}$ where [ ] ${ }^{T}$ represents the matrix transform operation. The conditional mean required in (6) is calculated using the joint probability distribution of the target and reference images. Equation (6) can further be expressed as:

$$
S(\mathrm{~m})=\sum_{i=1}^{N}\left(I_{i}-\hat{P}_{i}\right)^{2}
$$

where,

$$
\hat{P}_{i}=E\left(I_{i} \mid P_{i} \in \Delta_{k}\right)
$$

and $\Delta_{k}$ is the histogram bin which includes $\hat{P}_{i}$. Here, $\hat{P}_{i}$ is calculated using the joint probability distribution of $I$ and $P$, which makes the SCV similarity measure appropriate to use for multimodal registration applications. This similarity between two images is calculated at each iteration of the optimization process until either a threshold value of the maximum similarity between two images is found or another criteria, e.g., maximum number of iterations, has been reached.

\section{THE FAST REGISTRATION TECHNIQUE}

In this section, the new registration algorithm is described for the application of registering 3D CT data to $2 \mathrm{D}$ single plane fluoroscopy frames. The proposed approach simulates in-plane translations $\left(T_{x}\right.$ and $\left.T_{y}\right)$ and rotation $\left(R_{z}\right)$ in $2 \mathrm{D}$ instead of involving the full $3 \mathrm{D}$ volume at each iteration. For estimation of the out-of-plane rotation parameters $\left(R_{x}\right.$ and $\left.R_{y}\right)$ the proposed approach processes only a few selected slices from the 3D CT data. The iterative algorithm proceeds in three stages: in the first stage only in-plane parameters are updated, in the second stage all parameters except out-of-plane translation are updated, and all parameters are updated in the third stage. At the end of the second stage, the full 3D volume is updated once using the estimated rigid-body parameters. This update is then followed by DRR generation from the updated 3D volume and estimation of the out-of-plane translation $\left(T_{z}\right)$. Figure. 2 shows a block diagram of the proposed algorithm.

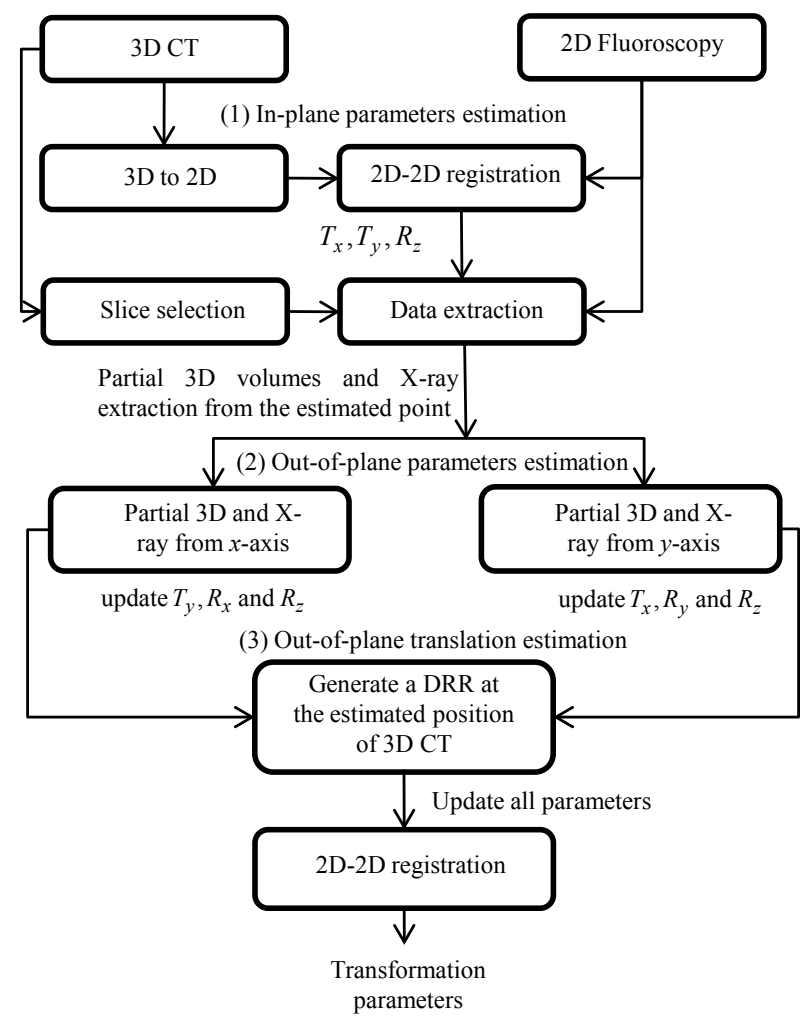

Figure 2: Block diagram of the proposed algorithm

To highlight the edges of the objects, we adopt the edgeenhancing approach proposed in (Pickering et al., 2009; 
Scarvell et al., 2010). Both the DRR and fluoroscopy images are filtered using progressively narrower Laplacian-ofGaussian (LoG) filters in each stage of the optimization process.

\subsection{DRR generation}

The DRR generation process involves projecting the 3D volume onto a 2D plane to produce 2D images that will be compared with the fluoroscopy images. This rendering process can be classified into software-based and hardware-based DRR generation techniques. Several hardware based techniques have been proposed in the literature (Bethune and Stewart, 2005; Cabral et al., 1995). These approaches require either an advanced graphics cards or specific hardware arrangements to accelerate the DRR generation process (Gendrin et al., 2012; Lacroute and Levoy, 1994).

Among the software based techniques, ray casting (Sherouse et al., 1990) is the most straightforward method, which enables generation of high quality DRRs but with significantly higher computational cost. To partially minimize the computational cost, other DRR generation techniques have been proposed in this category such as shear-warp factorization (Lacroute and Levoy, 1994), and attenuation field calculation (Russakoff et al., 2005). Though these DRR generation techniques produce high quality DRRs they are still rarely feasible for real-time applications.

An alternative, more efficient approach for DRR generation based on a projective transform has been proposed in (Pickering et al., 2009; Scarvell et al., 2010; Muhit et al., 2012). In this technique an assumption is made about the object's movement along the z-axis. As an object moves closer to the x-ray source it will produce a larger image on the image intensifier and vice-versa as shown in Figure. 3. This effect is modelled

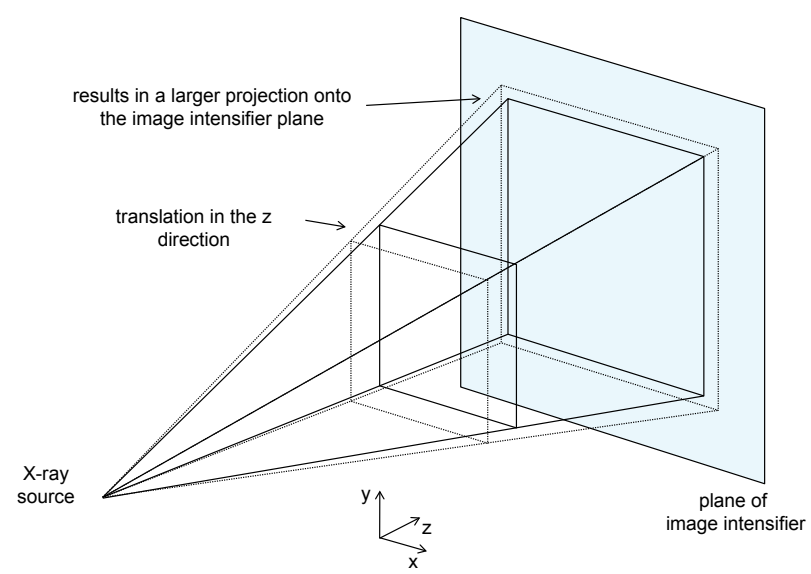

Figure 3: Illustration of the effect on the projection of the object's movement in the $z$ direction.

as a projective transformation applied to the CT data, and its thorough explanation is given in (Muhit et al., 2010).

In order to accurately estimate the scale change due to translation in the $z$ direction, the authors set up an experiment using a $400 \mathrm{~mm} \times 400 \mathrm{~mm}$ perspex calibration box. The front and back planes had tantalum beads in a regular grid pattern. The projective transformation parameter $W_{z}$ was calculated as follows:

$$
W_{z}=\frac{(a / b-1)}{d}
$$

where $d$ is the distance between the two parallel planes, $a$ is the distance between two beads in the plane closer to the x-ray source and $b$ for the other plane. The beads were placed equally spaced in both the planes but their projection differed due to the conical spread of the x-rays. The authors found that, for that specific fluoroscopy unit, the projective transformation parameter was equal to 0.0009 . This means that a movement of $1 \mathrm{~mm}$ (1 pixel) in the $z$ direction will correspond to a scale change of 0.0009. In this technique, after applying this projective transformation, a 2D projection was generated by simply summing up the voxel values along the $z$ direction. Our proposed algorithm generates the DRR based on this technique.

Since the proposed algorithm reduces the computational complexity by reducing the number of $3 \mathrm{D}$ volume updates at every iterative step this also reduces the number of times a DRR must be generated. Hence, employing fast DRR generation techniques would have little effect on the speedup of our proposed registration process.

\subsection{In-plane translations and rotation estimation}

Since we coarsely simulate the in-plane parameters prior to estimating the out-of-plane parameters as proposed in (Pickering et al., 2009), and the DRR is the projection of the voxels of the CT in the $z$ direction, translations in the $x$ and $y$ directions of the 3D CT volume will correspond to identical translations in the $x$ and $y$ directions for the 2D DRR. It is clear from (2) that rotation around the $z$-axis can also be applied directly in the $2 \mathrm{D}$ space.

To estimate the in-plane parameters, we generate a DRR from the original 3D CT volume and both the DRR and fluoroscopy images are filtered. Then, during the iterative loop, we update the fluoroscopy image prior to the LoG filter operation and compare with the filtered DRR. The proposed algorithm uses the Inverse Compositional Algorithm (ICA) proposed in (Baker and Matthews, 2001) as the optimization process for this in-plane parameter estimation stage. As long as the initial deformation remains inside the maximum limit, which has been described in result section, the proposed algorithm estimates a reasonable set of transformation parameters for the next steps. Since determining the true in-plane parameter values will depend on the estimated out-of-plane parameters, the proposed algorithm estimates these in-plane parameters at every stage of the registration procedure.

\subsection{Out-of-plane rotations estimation}

To track the out-of-plane rotations, the proposed algorithm decouples the rotation around the $x$-axis and rotation around the $y$-axis of the 3D volume. From equation (3) it can be easily understood that, when any object is rotating around the $x$-axis, the projection of the object in the 2D DRR will stretch or shrink along the $y$-axis depending on its depth in the $z$-direction. Similarly, from equation (4), the rotation of any object around the 
$y$-axis will stretch or shrink the object in the 2D DRR along the $x$-axis depending on its depth in the $z$-direction.

To estimate the rotation around the $x$-axis $\left(R_{x}\right)$ the proposed algorithm creates a partial 3D volume by selecting some effective slices in the $z-y$ plane. A set of columns are also extracted from the fluoroscopy image at the same position on the $x$-axis as the selected slices. Similarly, to estimate the rotation around the $y$-axis $\left(R_{y}\right)$ the proposed algorithm creates a partial 3D volume by selecting some effective CT slices in the $x$-z plane. A set of rows are also extracted from the fluoroscopy image at the same position on the $y$-axis as the selected slices.

The optimization procedure is then applied separately for rotation around the $x$ and $y$ axes. At every iteration of this stage the two partial 3D volumes are updated prior to summing in the $z$-direction and calculating the similarity measures with the selected $\mathrm{x}$-ray fluoroscopy data. As mentioned earlier, the inplane parameter estimation process depends on the current values of the out-of-plane parameters, so we fine-tune the $T_{y}$ and $R_{z}$ parameters along with the rotation around the $x$-axis $\left(R_{x}\right)$ during this stage. Similarly $T_{x}$ and $R_{z}$ are fine-tuned along with rotation around the $y$-axis $\left(R_{y}\right)$. The slice selection process is described in detail in the next section.

As mentioned previously, for rotations around the $x$ and $y$ axes, the object in the 2D DRR will stretch or shrink depending on its depth in the $z$ direction. To accurately simulate these

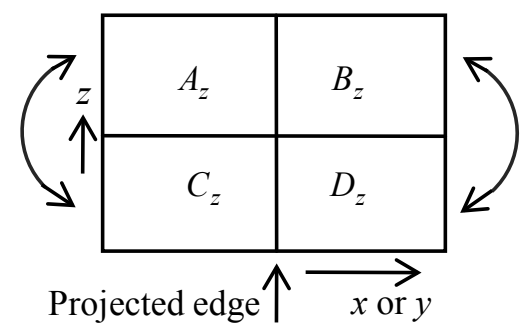

Figure 4: Demonstration of informative slice selection technique
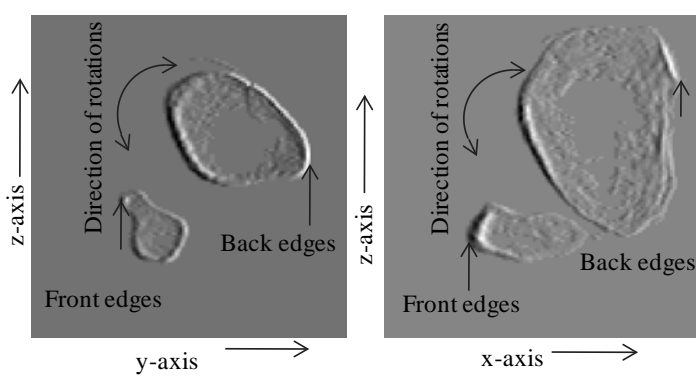

Figure 5: Demonstration of informative slice selection technique

rotations in 2D, we need an estimate of the $z$ coordinates of the edges of the selected CT slices from the full 3D volume. We adopt the following estimate from (Pickering et al., 2009).

$$
\hat{z}_{i}=\frac{\sum_{z} z\left|V_{e}\right|}{\sum_{z}\left|V_{e}\right|}
$$

In (10), $\hat{z}_{i}$ are estimates of the object edges in the $z$-direction and $V_{e}$ is a Laplacian-of-Gaussian filtered version of the partial 3D volume. Since we track out-of-plane rotations separately, we only filter the partial volumes and extracted fluoroscopy segments in one direction. When estimating the rotation around the $x$-axis we filter along the $y$-direction and when estimating the rotation around the $y$-axis we filter along the $x$-direction.

Since we only update and measure similarity on a small part of the CT volume and fluoroscopy images, our proposed approach is much faster than the approach which employs full 3D updates at every iterative step.

\subsubsection{Slice selection process}

To find the most informative slices, we look for edges at the front (closer to the X-ray source) and back (further away from the source) of the CT slices. Out-of-plane rotations introduce changes in the DRR and these changes depend on the position of the edges of the bones in the CT slices. The slices which have the largest gradients (strongest edges) either at the front or back of the slice will allow a more reliable estimate of the out-ofplane rotation. The proposed algorithm selects the slices which have strongest edges in diagonally opposite corners of the slices of the CT volume. To find the strongest edges we consider the magnitude of the gradient values of each individual slice. First we calculate the gradient of the volume in the $x$ and $y$ directions. Then we use the following method to find the most informative slices for out-of-plane rotation estimation.

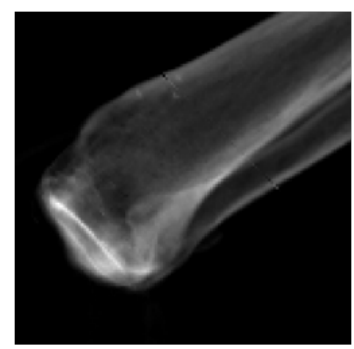

(a)

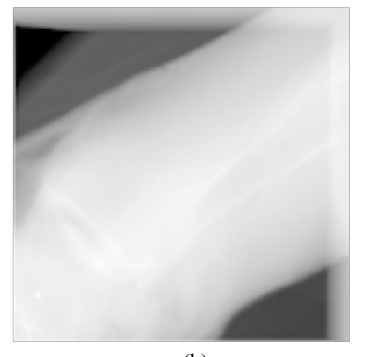

(b)
Figure 6: 2D DRR (a) and synthetic fluoroscopy frame (b) for Tibia

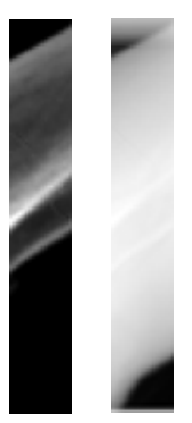

(a)

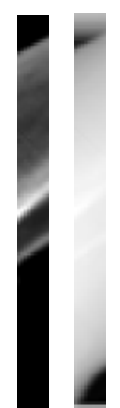

(b)

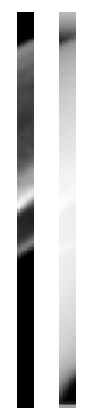

(c)
Figure 7: Partial DRR and extracted x-ray fluoroscopy columns for, (a) 20 slices, (b) 10 slices and (c) 5 slices to estimate $R_{x}$

Consider a slice of the gradient image, $I_{g}$. We first divide 


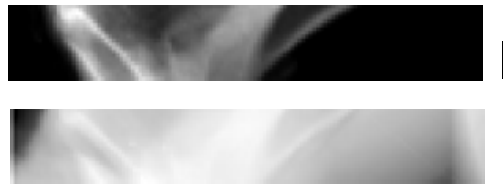

(a)

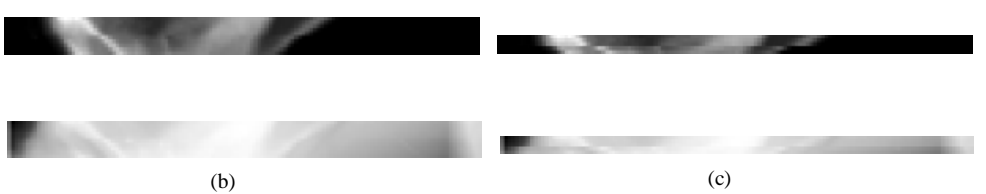

(b) (c)

Figure 8: Partial DRR and extracted x-ray fluoroscopy rows for, (a) 20 slices, (b) 10 slices and (c) 5 slices to estimate $R_{y}$

the slice into four quarters and sum the gradient values in each quarter. Let $A_{z}, B_{z}, C_{z}$ and $D_{z}$ denote the sum of the gradient values of each corner block as shown in Figure. 4 . When a DRR is generated by summing in the $z$-direction, the effect of out-ofplane rotations will be to stretch or shrink the distance between edges at the front and back of the 3D volume. From Figure. 5 it is clear that when a slice rotates clockwise the effect will be to increase the distance between the projection of the edges at the front left and back right of the slice. Similarly when the slice rotates anti-clockwise the effect will be to decrease the distance between the projection of the edges at the front left and back right of the slice. So the slices which have strong edges at diagonally opposite corners will provide the most reliable estimate of the out-of-plane rotation of the volume.

To measure these diagonally opposite gradient magnitudes we simply use the following formula:

$$
\begin{aligned}
& \hat{G}_{a \rightarrow d}=A_{z}+D_{z} \\
& \hat{G}_{c \rightarrow b}=C_{z}+B_{z}
\end{aligned}
$$

where $\hat{G}_{a \rightarrow d}$ and $\hat{G}_{c \rightarrow b}$ are the sum of the absolute values of diagonally opposite gradient magnitudes. To select the informative slices we compare these summed gradient magnitudes and choose the slices which have the largest values. This selection process is done for both $x$ and $y$ directions independently. Figure. 7 and Figure. 8 show examples of the informative slices selected from the DRR and fluoroscopy images shown in Figure. 6. In the experimental results section we will show that as few as 5 slices can be used to estimate the out-of plane rotations.

\subsection{Out-of-plane translation estimation}

From equation (1) it can be understood that translations in the $x$ and $y$ directions of the 3D CT volume will correspond to identical translations in the $x$ and $y$ directions for the 2D DRR. However, translation in the $z$ direction is different. As shown in Figure. 3, the x-ray beams in the fluoroscopy unit diverge from a common point as they pass through the object and arrive at the image intensifier. Consequently, as an object moves closer to the x-ray source, it will produce a larger image at the image intensifier. This means that translation in the $z$ direction will result in a change in scale of the object in the fluoroscopy frame. Hence, the out-of-plane translation can be estimated by measuring the small amount of zoom that must be applied to the DRR to register it with the fluoroscopy frame. This change in scale is usually difficult to measure precisely as a large translation in the $z$ direction will result in only a very small scale change in the fluoroscopy frame.
In our proposed algorithm we estimate this out-of-plane translation parameter $\left(T_{z}\right)$ by updating the $2 \mathrm{D}$ DRR at every iteration instead of updating the full 3D volume. As in (Scarvell et al., 2010), we simulate the small zoom factor on the 2D DRR by applying equal scaling in both $x$ and $y$ directions. At the beginning of this out-of-plane parameter estimation stage we generate a DRR and estimate $z_{i}$ once using the full 3D volume at the $3 \mathrm{D}$ position estimated at the last iteration of the second stage. Since we fine-tune the other five parameters in this stage as well, we need to estimate depth in the $z$ direction for out-ofplane rotation parameters. Since we have already estimated the out-of-plane rotations in the second stage and the parameters are already very close to the true position it is assumed that, for small out-of-plane rotations, the position of the object edges in the $z$ direction remain nearly constant and hence we can use the estimated $z_{i}$ values during the remaining $2 \mathrm{D}$ updates. To summarizing the above, the proposed algorithm proceeds as follows:

\section{In-plane parameters estimation:}

Pre-compute:

1. Construct the DRR image of the original CT volume (Summing up voxel values in $z$ direction after applying projective transformation).

Iterate the following steps:

1. Apply a 2D transformation on the fluoroscopy frame.

2. Apply LoG filtering to both the DRR and fluoroscopy frames to highlight the edges of the object.

3. Update the in-plane transformation parameters $\left(T_{x}, T_{y}\right.$ and $R_{z}$ ) using the SCV similarity measure.

4. Repeat the algorithm with the updated parameters until the maximum number of iteration is reached.

Out-of-plane rotations estimation with fine-tuning of the estimated in-plane parameters:

Pre-compute:

1. Select informative slices by using the above-mentioned slice selection technique.

2. Extract partial 3D volumes for the $x$ and $y$-axis separately and extract partial fluoroscopy frames from the same positions where the CT slices are extracted.

Iterate the following steps: 
1. Apply 3D volume transformations on the partial 3D volumes separately and generate DRRs.

2. Apply one dimensional LoG filtering to the partial DRRs and corresponding fluoroscopy rows and columns.

3. Update corresponding transformation parameters using the SCV similarity measure (e.g. for the partial volume extracted from the $x$-axis, the parameters $T_{y}, R_{x}$ and $R_{z}$ are updated and, for the partial volume extracted from the $y$ axis, the parameters $T_{x}, R_{y}$ and $R_{z}$ are updated).

4. Repeat the algorithm with the updated transformation parameters until the maximum number of iterations is reached.

Out-of-plane translation estimation with fine-tuning of the other five parameters:

Pre-compute:

1. Apply a 3D transform on the CT volume and construct a DRR image using the estimated position from the out-of plane parameters estimation step.

2. Estimate the value of $\hat{z}_{i}$ by using equation (10).

Iterate the following steps:

1. Apply a 2D transform on the DRR image.

2. Apply two dimensional LoG filtering to both the DRR and fluoroscopy frame to highlight the edges of the object.

3. Update all the six transformation parameters using the SCV similarity measure.

4. Repeat the algorithm with the updated parameters until the maximum number of iterations is reached.

\section{EXPERIMENTAL RESULTS}

In this section, the speed, accuracy and success rate of an algorithm which performs full 3D updates at each iteration is compared with the same algorithm but with the proposed slicebased approach adopted. The 3D-2D registration algorithm used for this comparison is the algorithm presented in (Pickering et al., 2009) which was shown to provide superior performance for 3D-2D registration of CT to fluoroscopy in terms of accuracy and registration success rate.

In the remainder of this paper, the original algorithm presented in (Pickering et al., 2009) which employs full 3D updates at every iteration will be referred to as the full $3 \mathrm{D}$ algorithm and the fast version of this algorithm which adopts the estimation of in-plane parameters by using simple 2D transformations instead of 3D transformations and the slice-based approach for out-of-plane rotation estimation will be referred to as the proposed slice-based algorithm.

These two algorithms were used to register a segmented 3D CT volume of a female tibia and femur to synthetic $2 \mathrm{D}$ fluoroscopy images and also to real fluoroscopy data of the same bone. To reduce memory requirements, lower resolution versions of the original data were used for this experiment with a resolution of 1 pixel equal to $1 \mathrm{~mm}$.

\subsection{Experimental results with synthetic fluoroscopy data}

For the first experiment, synthetic fluoroscopy images were generated from the 3D CT data using a logarithmic attenuation function on the sum of the voxel values of the unsegmented CT data along rays that simulate the path of a point source of x-rays. The original 3D position of the CT that was used to produce the synthetic fluoroscopy image was known and was used as the gold standard to evaluate the registration performance. A synthetic fluoroscopy frame created from an unsegmented CT and a DRR generated using the same but segmented CT is shown in Figure. 6. Test volumes were generated from the original 3D CT data using uniformly distributed random values for the six motion parameters $( \pm 5 \mathrm{~mm}$ translations and \pm 5 degrees of rotation).

For a robust comparison, the registration was performed for 100 such random starting positions. The algorithms under investigation were then used to register the transformed CT images to the fluoroscopy image. Although the initial starting positions were chosen randomly, they were the same for both algorithms. For each starting position, the algorithms are allowed to make 45 iterations as our results showed that this number of iterations was enough to estimate the 3D pose of a bone. For fair comparison both the algorithms were set to follow the same fashion such as among these 45 iterations, 21 iterations were used in the first in-plane parameters estimation stage, 7 iterations were used to estimate out-of-plane rotations and the remaining iterations were used to estimate the out-of-plane translation with fine-tuning of the other five parameters. To evaluate the registration performance in physical space, the maximum registration error (MRE) measure is used which is given by:

$$
\operatorname{MRE}=\max _{i}\left(\sqrt{\left(x_{i}^{\prime}-x_{i}\right)^{2}+\left(y_{i}^{\prime}-y_{i}\right)^{2}+\left(z_{i}^{\prime}-z_{i}\right)^{2}}\right)
$$

where $\left(x_{i}, y_{i}, z_{i}\right)$ are the positions of the $i$ th voxel in the reference volume and $\left(x_{i}^{\prime}, y_{i}^{\prime}, z_{i}^{\prime}\right)$ are the positions of the $i$ th voxel in the target volume. If the value of MRE for the final iteration was greater than $1 \mathrm{~mm}$ then the algorithm was considered to have failed to register the two images.

Since the original true 3D position corresponded to zero translation and rotation, the registration goal was to produce transform parameters as close to zero as possible. Therefore, to evaluate the registration performance in parameter space, the registration error was defined by the root mean square (RMS) of the transform parameter values for translations and rotations using the following equations:

$$
\begin{aligned}
& E_{t}=\frac{\sqrt{T_{x}^{2}+T_{y}^{2}+T_{z}^{2}}}{3} \\
& E_{r}=\frac{\sqrt{R_{x}^{2}+R_{y}^{2}+R_{z}^{2}}}{3}
\end{aligned}
$$

where $E_{t}$ and $E_{r}$ are the registration errors for the translation and rotation parameters, respectively. The average RMS error at each iteration for all successful cases is shown in Figure. 9. The graphs in Figure. 9 were generated using bi-cubic interpolation in all algorithms. For the slice based approaches (20 
Table 1: Mean and Standard Deviation (SD) of the Transform Parameter Errors for Synthetic Fluoroscopy Data

\begin{tabular}{|l|c|c|c|c|c|c|c|}
\hline \hline \multirow{3}{*}{ Bone } & \multirow{2}{*}{ Algorithms } & \multicolumn{2}{|c|}{ Mean/SD for translations (mm) } & \multicolumn{2}{c|}{ Mean/SD for rotations (degrees) } \\
\cline { 3 - 8 } & & $T_{x}$ & $T_{y}$ & $T_{z}$ & $R_{x}$ & $R_{y}$ & $R_{z}$ \\
\hline \multirow{5}{*}{ Tibia } & 5 Slices & $0.01 / 0.01$ & $-0.12 / 0.00$ & $0.00 / 0.08$ & $0.04 / 0.03$ & $-0.24 / 0.05$ & $-0.00 / 0.00$ \\
& 10 Slices & $0.00 / 0.01$ & $-0.12 / 0.00$ & $0.00 / 0.07$ & $0.04 / 0.02$ & $-0.25 / 0.03$ & $-0.00 / 0.00$ \\
& 15 Slices & $0.01 / 0.00$ & $-0.13 / 0.00$ & $0.02 / 0.09$ & $0.06 / 0.02$ & $-0.28 / 0.02$ & $-0.00 / 0.00$ \\
& 20 Slices & $0.01 / 0.01$ & $-0.13 / 0.00$ & $-0.00 / 0.12$ & $0.08 / 0.02$ & $-0.28 / 0.02$ & $-0.00 / 0.00$ \\
& Full 3D & $0.02 / 0.00$ & $-0.15 / 0.00$ & $0.02 / 0.02$ & $0.11 / 0.00$ & $-0.34 / 0.00$ & $-0.01 / 0.00$ \\
\hline \multirow{5}{*}{ Femury } & 5 Slices & $0.06 / 0.01$ & $0.00 / 0.01$ & $-0.33 / 0.13$ & $0.20 / 0.14$ & $0.04 / 0.17$ & $0.05 / 0.00$ \\
& 10 Slices & $0.05 / 0.01$ & $0.00 / 0.01$ & $-0.27 / 0.12$ & $0.14 / 0.05$ & $0.11 / 0.11$ & $0.04 / 0.00$ \\
& 15 Slices & $0.06 / 0.01$ & $0.00 / 0.01$ & $-0.28 / 0.12$ & $0.15 / 0.06$ & $0.04 / 0.10$ & $0.04 / 0.00$ \\
& 20 Slices & $0.06 / 0.01$ & $0.00 / 0.01$ & $-0.23 / 0.08$ & $0.24 / 0.07$ & $0.03 / 0.09$ & $0.03 / 0.00$ \\
& Full 3D & $0.00 / 0.00$ & $-0.03 / 0.00$ & $-0.26 / 0.01$ & $0.19 / 0.00$ & $0.03 / 0.00$ & $0.07 / 0.00$ \\
\hline \hline
\end{tabular}

slices, 15 slices, 10 slices and 5 slices) the in-plane parameters and out-of-plane translation parameter estimation stages are the same. The only difference is the number of CT slices used in the out-of-plane rotation estimation stage. From Figure. 9 it can be seen that the final average RMS errors for the slice based approaches converge to an average value which is close to that of the full 3D algorithm. The main difference is that the convergence rate for the out-of-plane parameters depends on the number of CT slices used to estimate them.

The final estimation error for the out-of-plane parameters estimation stage also impacts the convergence of the registration algorithms. The average registration error of the proposed algorithm was similar to that of the full 3D algorithm for both the in-plane and out-of-plane parameters. The in-plane translation error was between 0.1 and $0.2 \mathrm{~mm}$ and for in-plane rotation the error was $0.1-0.15$ degrees. The out-of-plane rotation error was 0.2-0.35 degrees and the out-of-plane translation error was 0.2-0.4 mm. A comparison of the mean and standard deviation (SD) of the proposed slice based algorithms and the full 3D algorithm is shown in Table 1. From Table 1 we can see that the slice based algorithms provide results similar to each other and close to that of the full 3D algorithm. These results also show that the accuracy improves as more slices are used.

The next performance measure used to evaluate the registration algorithms is the number of starting positions for which the algorithms successfully registered the displaced volumes. This measure is referred to as the success rate of the algorithms. The percentage of the 100 initial starting positions for which each algorithm successfully registered the displaced $3 \mathrm{D}$ volumes is presented in Table 2. From Table 2 it can be seen that the success rate of the slice based approaches also increases with the number of CT slices used. These results show that the full 3D, 20 slice, 15 slice and 10 slice algorithms provide the highest success rate of almost $100 \%$ for the tibia and $95 \%$ for the femur. Alternatively the algorithm which uses only $5 \mathrm{CT}$ slices to track the out-of-plane rotations shows a lower success rate. It is understood from the failure cases that all positions for which the proposed algorithm failed contained a parameter which had a value close to \pm 5 . This indicates that the limit to the capture range of the algorithm is approximately \pm 4.5 pixels of translation and \pm 4.5 degrees of rotation. This range could possibly
Table 2: Success Rate of the Registration Algorithms for Synthetic Fluoroscopy Data

\begin{tabular}{l|c|c}
\hline \hline Algorithms & Tibia & Femur \\
\hline 5 Slices & $97 \%$ & $92 \%$ \\
10 Slices & $100 \%$ & $95 \%$ \\
15 Slices & $100 \%$ & $95 \%$ \\
20 Slices & $100 \%$ & $95 \%$ \\
Full 3D & $100 \%$ & $95 \%$ \\
\hline \hline
\end{tabular}

be extended by using more coarse stages with larger LoG filter kernels.

Figure. 10 shows a bar graph plot of the average CPU time required to register one displaced $3 \mathrm{D}$ volume when using each algorithm. These results were obtained with MATLAB 7.8 in an Intel(R) Core(TM) 2 Duo CPU E6750 at $2.66 \mathrm{GHz}$ with $1.99 \mathrm{~GB}$ of RAM. For these results the total number of iterations was set to 45 . The CPU time required for the different slice based algorithms are approximately 10.3, 12.5, 15.4 and 18.9 times faster than the full 3D algorithm for 20, 15, 10 and 5 CT slices respectively.

\subsection{Experimental results using real fluoroscopy data}

Next, a similar experiment to that described in the previous section was performed but with real fluoroscopy images. In this experiment, cadaveric lower limb specimens were used and the gold standard 3D position of the bones was measured using Roentgen Stereo Analysis (RSA) (Selvik, 1989). 230 mm Steinman pins were inserted about $150 \mathrm{~mm}$ proximal and 150 $\mathrm{mm}$ distal to the lateral epicondyle of the femur, in the coronal plane. The lower limbs were then placed in a purpose-built transparent Perspex cage, as shown in Figure. 11.

In this fixed position, a fluoroscopy frame was captured for $3 \mathrm{D}-2 \mathrm{D}$ registration. Two orthogonal x-rays were also captured using the calibrated grid specially designed for RSA. To compare the full 3D algorithm and the proposed slice-based approach, a similar experiment was performed to that described in the previous section. The 3D position of the bones measured by RSA was used as the gold standard to evaluate the registration performance. Test volumes were generated from the $3 \mathrm{D}$ CT data using uniformly distributed random values for the six 


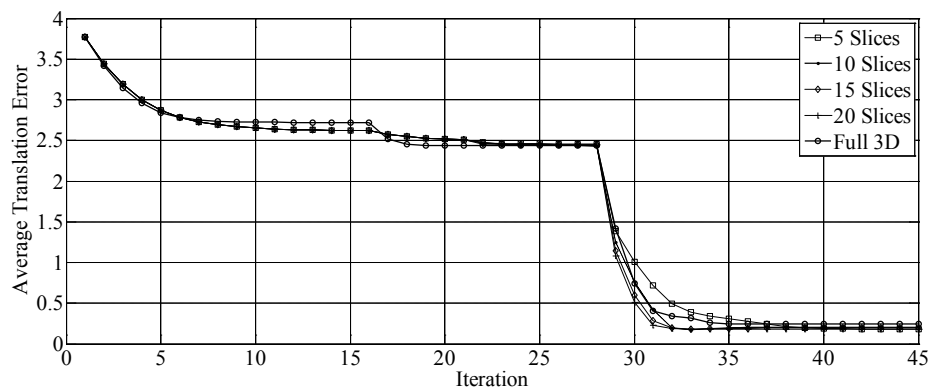

(a)

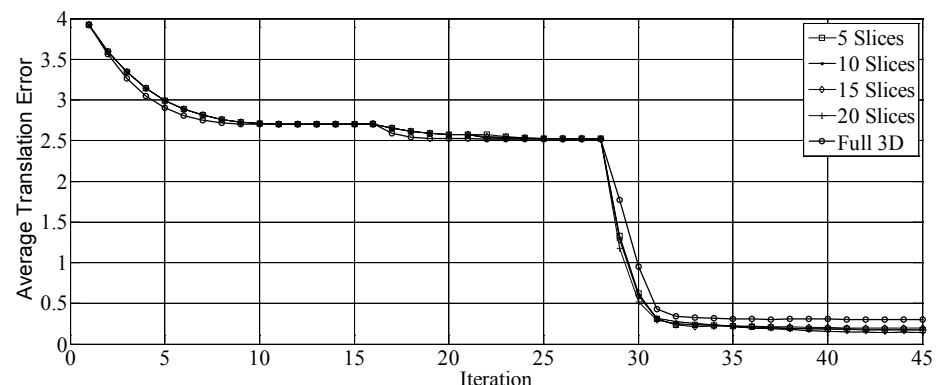

(b)

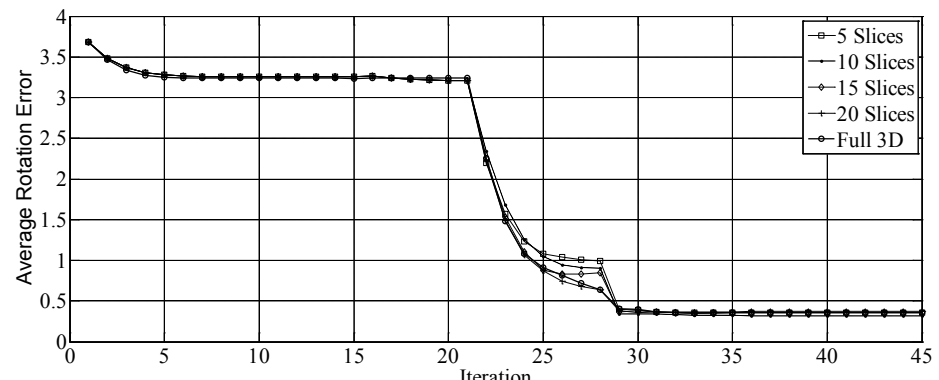

(c)

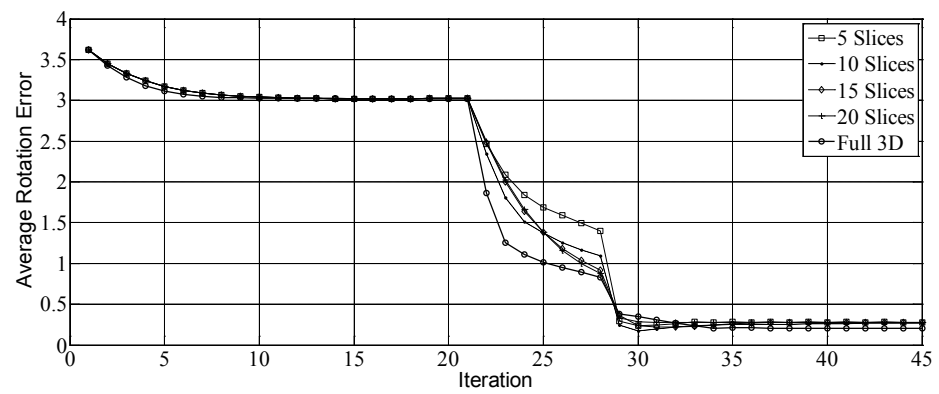

(d)

Figure 9: Average translation and rotation errors at each iteration for all the cases where the algorithms successfully converged for synthetic fluoroscopy data (a) translation error (tibia), (b) translation error (femur), (c) rotation error (tibia), and (d) rotation error (femur).

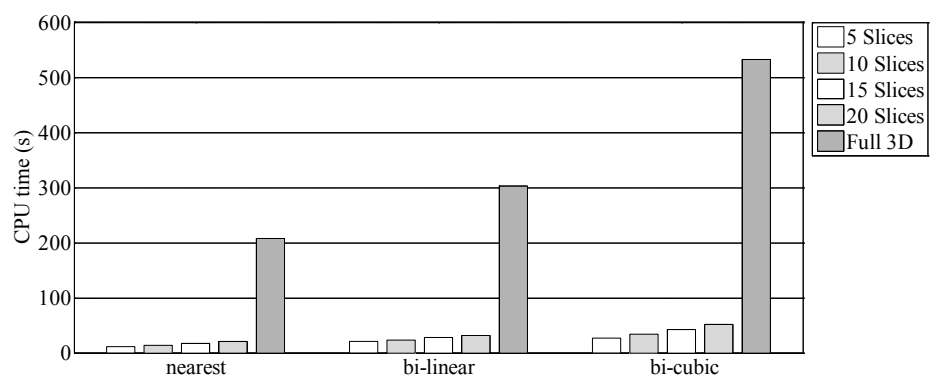

Figure 10: Average CPU time required by the algorithms tested to register a 3D CT volume $(128 \times 128 \times 128)$ when using nearest-neighbour, bi-linear, and bi-cubic interpolation. 


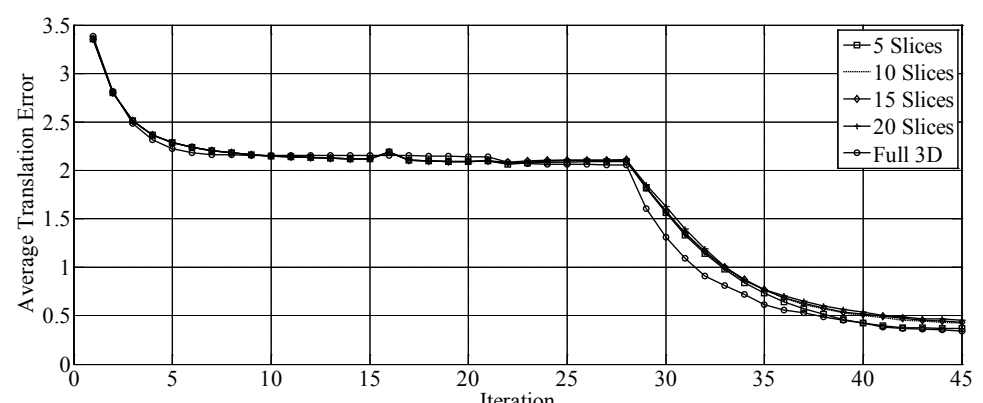

(a)

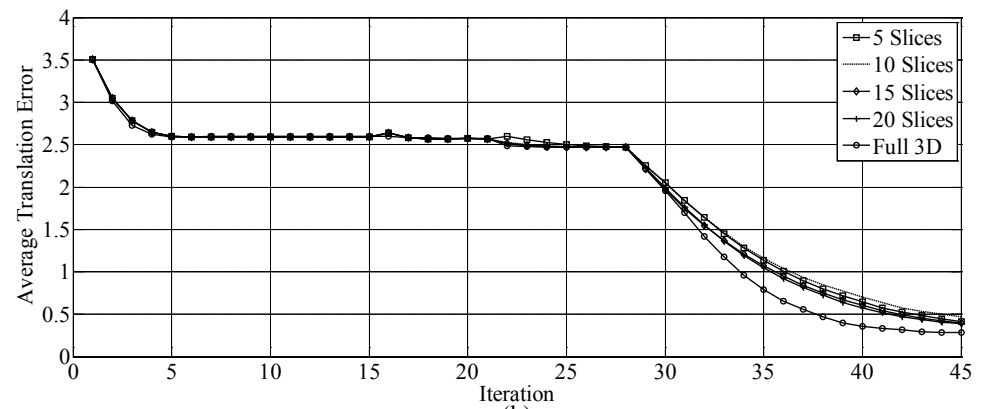

(b)

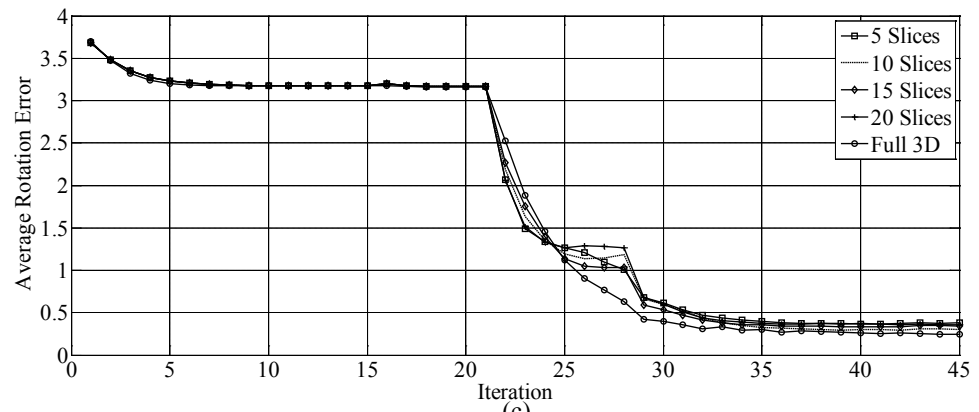

(c)

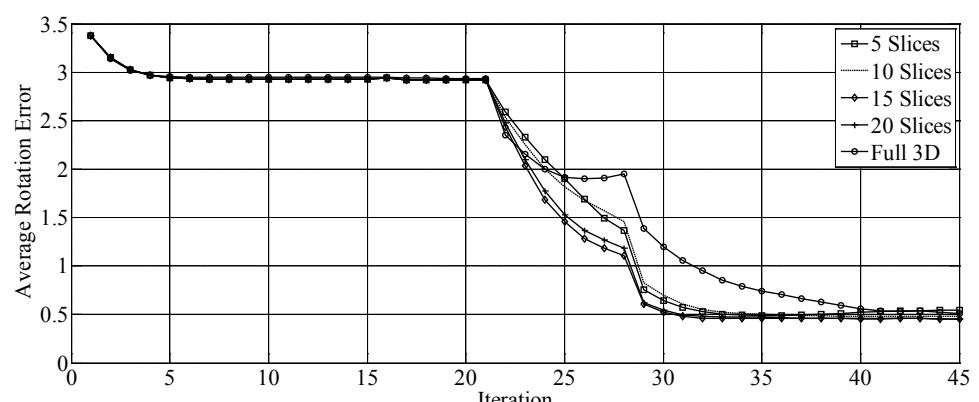

(d)

Figure 12: Average translation and rotation errors at each iteration for all the cases where the algorithms successfully converged for real fluoroscopy data (a) translation error (tibia), (b) translation error (femur), (c) rotation error (tibia), and (d) rotation error (femur). 
Table 3: Mean and Standard Deviation (SD) of the Transform Parameter Errors for Real Fluoroscopy Data

\begin{tabular}{|l|c|c|c|c|c|c|c|}
\hline \hline \multirow{2}{*}{ Bone } & \multirow{2}{*}{ Algorithms } & \multicolumn{3}{|c|}{ Mean/SD for translations (mm) } & \multicolumn{2}{c|}{ Mean/SD for rotations (degrees) } \\
\cline { 3 - 8 } & & $T_{x}$ & $T_{y}$ & $T_{z}$ & $R_{x}$ & $R_{y}$ & $R_{z}$ \\
\hline \multirow{5}{*}{ Tibia } & 5 Slices & $0.41 / 0.01$ & $-0.31 / 0.01$ & $0.74 / 0.32$ & $-0.48 / 0.23$ & $-0.30 / 0.19$ & $0.04 / 0.03$ \\
& 10 Slices & $0.41 / 0.01$ & $-0.31 / 0.02$ & $0.69 / 0.41$ & $-0.45 / 0.22$ & $-0.36 / 0.16$ & $0.02 / 0.03$ \\
& 15 Slices & $0.41 / 0.01$ & $-0.31 / 0.02$ & $0.67 / 0.41$ & $-0.45 / 0.23$ & $-0.38 / 0.18$ & $0.03 / 0.04$ \\
& 20 Slices & $0.39 / 0.01$ & $-0.33 / 0.01$ & $0.34 / 0.29$ & $-0.44 / 0.23$ & $-0.31 / 0.14$ & $0.04 / 0.02$ \\
& Full 3D & $0.39 / 0.02$ & $-0.36 / 0.02$ & $0.64 / 0.24$ & $-0.41 / 0.13$ & $-0.52 / 0.15$ & $0.14 / 0.02$ \\
\hline \multirow{5}{*}{ Femury } & 5 Slices & $0.37 / 0.02$ & $-0.40 / 0.05$ & $-0.44 / 0.34$ & $0.49 / 0.31$ & $-0.06 / 0.30$ & $0.30 / 0.05$ \\
& 10 Slices & $0.37 / 0.02$ & $-0.40 / 0.04$ & $-0.56 / 0.42$ & $0.47 / 0.21$ & $-0.27 / 0.20$ & $0.30 / 0.05$ \\
& 15 Slices & $0.37 / 0.02$ & $-0.41 / 0.03$ & $-0.56 / 0.33$ & $0.37 / 0.16$ & $-0.23 / 0.24$ & $0.27 / 0.05$ \\
& 20 Slices & $0.37 / 0.02$ & $-0.41 / 0.03$ & $-0.56 / 0.35$ & $0.37 / 0.14$ & $-0.23 / 0.23$ & $0.28 / 0.05$ \\
& Full 3D & $0.36 / 0.01$ & $-0.42 / 0.02$ & $-0.65 / 0.24$ & $0.28 / 0.12$ & $-0.42 / 0.13$ & $0.21 / 0.08$ \\
\hline \hline
\end{tabular}

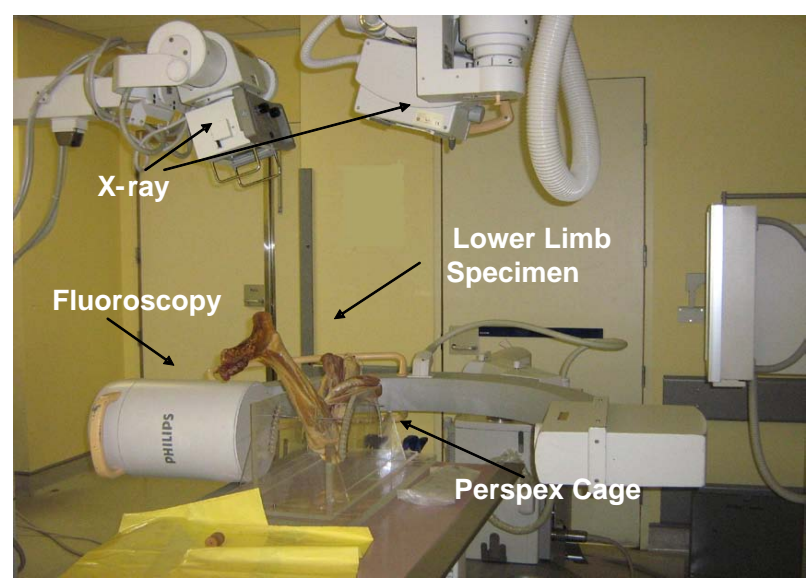

Figure 11: Simultaneous Fluoroscopy and RSA experimental setup using cadaver lower limb specimens.

transform parameters $( \pm 5 \mathrm{~mm}$ translations and \pm 5 degrees of rotation) relative to the gold standard position. Hence, a value of zero for all parameters corresponded to the 3D position measured by RSA. The random starting positions that were used for the synthetic data were also used in this experiment for real data.

For each test volume, the registered 3D position of the bones was then estimated using the full 3D and sliced based registration algorithms and compared with the gold standard position provided by RSA. The average RMS error at each iteration for all successful cases is shown in Figure. 12 for the real fluoroscopy data. The in-plane translation error for this real fluoroscopy data was between 0.2 to $0.4 \mathrm{~mm}$ and for in-plane rotation the error was 0.2-0.4 degrees. The out-of-plane rotation error was 0.3-0.5 degrees and the out-of-plane translation error was $0.3-0.7 \mathrm{~mm}$. The mean and standard deviation (SD) of the final registration error for the proposed slice based algorithms and the full 3D algorithm for real fluoroscopy data is shown in Table 3. From Table 3 we can see that the slice based algorithms provide quite similar results to that of the full 3D algorithm.

The percentage of the 100 initial starting positions for which each algorithm successfully registered the displaced 3D volumes is presented in Table 4. These results show that the full
Table 4: Success Rate of the Registration Algorithms for Real Fluoroscopy Data

\begin{tabular}{l|c|c}
\hline \hline Algorithms & Tibia & Femur \\
\hline 5 Slices & $91 \%$ & $87 \%$ \\
10 Slices & $92 \%$ & $90 \%$ \\
15 Slices & $95 \%$ & $93 \%$ \\
20 Slices & $99 \%$ & $93 \%$ \\
Full 3D & $99 \%$ & $93 \%$ \\
\hline \hline
\end{tabular}

3D, 20 slice and 15 slice algorithms provide the highest success rate of almost $95 \%$ for the Tibia and $93 \%$ for the Femur. Alternatively, the algorithms which use only 10 or 5 CT slices to track the out-of-plane rotations showed lower success rates.

From the analysis of both real and synthetic fluoroscopy data, we can see that as few as 10 slices out of the original 128 slices can be used to estimate the out-of-plane rotation parameters. The proposed algorithm with 10 slices gives a speed-up factor of around 15.4 times compared with the algorithm which employs full 3D updates at every iteration.

\section{Conclusion}

This paper proposes a new low complexity 3D CT to single plane X-ray fluoroscopy image registration technique using the SCV multi-modal similarity measure and gradient based optimization. The unique features of the proposed algorithm are: the estimation of in-plane parameters by using simple 2D transformations instead of 3D transformations and the estimation of the out-of-plane rotations by using a small number of informative CT slices. Our experimental results show that the registration precision and success rate of our proposed algorithm is comparable to the algorithm employing full 3D volume updates at every iteration. Furthermore, using this proposed multi-phase registration approach, we achieve a speed-up factor of between 10 and 20 with no significant loss of accuracy.

\section{References}

Baker, S., Matthews, I., 2001. Equivalence and efficiency of image alignment algorithms. In: Proceedings of IEEE Conf. on Computer Vision and Pattern Recognition (CVPR). pp. 1090-1097. 
Bethune, C., Stewart, A. J., 2005. Adaptive slice geometry for hardwareassisted volume rendering. Journal of Graphics, GPU and Game Tools 10, 55-70.

Birkfellner, W., Seemann, R., et al., 2005. Fast DRR generation for 2D/3D registration. In: Medical Image Computing and Computer-Assisted Intervention-MICCAI. pp. 960-967.

Birkfellner, W., et al., 2003. A faster method for 3D/2D medical image registration-a simulation study. Physics in medicine and biology 48, 26652679.

Cabral, B., et al., 1995. Accelerated volume rendering and tomographic reconstruction using texture mapping hardware. In: Proceedings Volume Visualization Workshop, ACM SIGGRAPH. pp. 91-98.

Chen, X., et al., 2008. A computationally efficient method for automatic registration of orthogonal X-ray images with volumetric CT data. Physics in medicine and biology 53, 967-983.

de Bruin, P. W., et al., 2008. Image-based RSA: Roentgen stereophotogrammetric analysis based on 2D-3D image registration. Journal of Biomechanics 41, $155-164$.

Fu, D., Kuduvalli, G., 2008. A fast, accurate, and automatic 2D-3D image registration for image-guided cranial radiosurgery. Medical physics 35, 2180 2194.

Gendrin, C., et al., 2012. Monitoring tumor motion by real time 2D/3D registration during radiotherapy. Radiotherapy and Oncology 102 (2), 274-280.

Jans, H. S., et al., 2006. 3D interfractional patient position verification using 2D-3D registration of orthogonal images. Medical physics 33, 1420.

Lacroute, P., Levoy, M., July 24-29 1994. Fast volume rendering using a shearwarp factorization of the viewing transformation. In: Proceedings of SIGGRAPH). Orlando, FL, pp. 451-458.

Lavallee, S., Szeliski, R., 1995. Recovering the position and orientation of freeform objects from image contours using 3D distance maps. IEEE Transactions on Pattern Analysis and Machine Intelligence 17, 378-390.

Livyatan, H., et al., 2003. Gradient-based 2-D/3-D rigid registration of fluoroscopic X-ray to CT. IEEE Transactions on Medical Imaging 22, 1395-1406.

Lujan, A. E., et al., 1998. Determination of rotations in three dimensions using two-dimensional portal image registration. Medical physics 25, 703.

Maes, F., et al., 1999. Comparative evaluation of multiresolution optimization strategies for multimodality image registration by maximization of mutual information. Medical Image Analysis 3, 373-386.

Mahfouz, M. R., et al., 2003. A robust method for registration of threedimensional knee implant models to two-dimensional fluoroscopy images. IEEE Transactions on Medical Imaging 22, 1561-1574

Markelj, P., Tomaževič, D., Likar, B., Pernuš, F., 2012. A review of 3D/2D registration methods for image-guided interventions. Med. Imag. Anal. 16 (3), 642-661.

Markelj, P., et al., 2008. Robust gradient-based 3-D/2-D registration of CT and MR to X-ray images. IEEE Transactions on Medical Imaging 27, 1704 1714.

Muhit, A. A., Pickering, M., et al., August 31-September 4, 2010. A comparision of the 3D kinematic measurements obtained by single-plane 2D-3D image registration and RSA. In: Proc. IEEE 32nd Annual International Con- ference of the EMBS. Buenos Aires, Argentina, pp. 6288 - 6291.

Muhit, A. A., et al., 2012. A new multimodal similarity measure for the prospective kinematic analysis of knee joints. Journal of Bone and Joint Surgery-British Volume 94-B (SUPP XXII:101).

Penney, G. P., et al., 1998. A comparison of similarity measures for use in 2-D3-D medical image registration. IEEE Transactions on Medical Imaging 17, 586-595.

Pickering, M. R., et al., September 2-6, 2009. A new multi-modal similarity measure for fast gradient-based 2D-3D image registration. In: in 31st Annual International Conference of the IEEE EMBS. Minneapolis, Minnesota, USA, pp. 5821-5824.

Pluim, J. P. W., et al., 2003. Mutual-information-based registration of medical images: a survey. IEEE Transactions on Medical Imaging 22, 986-1004.

Russakoff, D. B., et al., 2005. Fast generation of digitally reconstructed radiographs using attenuation fields with application to 2D-3D image registration. IEEE Transactions on Medical Imaging 24, 1441-1454.

Scarvell, J. M., et al., 2010. New registration algorithm for determining 3D knee kinematics using CT and single plane fluoroscopy with improved out of plane translation accuracy. Journal of Orthopaedic Research 28, 334-340.

Selvik, G., 1989. Roentgen stereophotogrammetry. a method for the study of the kinematics of the skeletal system. Acta Orthopaedica Scandinavica Supplementum (232), 1-51.

Sherouse, G. W., et al., 1990. Computation of digitally reconstructed radiographs for use in radiotherapy treatment design. International Journal of Radiation Oncology Biology Physics 18, 651-658.

Tomazevic, D., et al., 2003. 3-D/2-D registration of CT and MR to X-ray images. IEEE Transactions on Medical Imaging 22, 1407-1416.

Wang, F., Vemuri, B. C., 2007. Non-rigid multi-modal image registration using cross-cumulative residual entropy. International journal of computer vision 74, 201-215.

Weese, J., et al., March 19-22 1997a. An approach to 2D/3D registration of a vertebra in 2D X-ray fluoroscopies with 3D CT images. In: Proceedings of (CVRMed/MRCAS). Grenoble, France, pp. 119-128.

Weese, J., et al., 1997b. Voxel-based 2-D/3-D registration of fluoroscopy images and CT scans for image-guided surgery. IEEE Transactions on Medical Imaging 1, 284-293.

Weese, J., et al., 1999. Fast voxel-based 2D/3D registration algorithm using a volume rendering method based on the shear-warp factorization. In: Proceedings of SPIE). Vol. 3661. pp. 802-810.

Yamazaki, T., Watanabe, T., Nakajima, Y., et al., 2004. Improvement of depth position in 2-D/3-D registration of knee implants using single-plane fluoroscopy. IEEE Transactions on Medical Imaging 23, 602-612.

Zollei, L., et al., 2001. 2D-3D rigid registration of X-ray fluoroscopy and CT images using mutual information and sparsely sampled histogram estimators. In: Proc. IEEE Int. Conf. on Computer Vision and Pattern Recognition). Los Alamitos, CA:, pp. 696-703.

Zuffi, S., Leardini, A., Catani, F., et al., 1999. A model-based method for the reconstruction of total knee replacement kinematics. IEEE Transactions on Medical Imaging 18, 981-991. 